\title{
Bullous Systemic Lupus Erythematosus and Membranous Lupus Nephritis in a Filipino Child
}

\author{
Marc Andrew O. Perez, ${ }^{1}$ Candice B. Brillante, ${ }^{1}$ Lourdes Paula R. Resontoc, ${ }^{1}$ \\ Dolores D. Bonzon, ${ }^{1}$ Francisco E. Anacleto, ${ }^{1}$ Eric T. Aragon,1 Cherica A. Tee, ${ }^{2}$ Sherbeth Mae M. Rey, ${ }^{2}$ \\ Georgina C. Pastorfide, ${ }^{3}$ Cybill Dianne C. Uy ${ }^{3}$ and Jolene Kristine G. Gatmaitan ${ }^{3}$ \\ ${ }^{1}$ Section of Nephrology, Department of Pediatrics, College of Medicine and Philippine General Hospital, University of the Philippines Manila \\ ${ }^{2}$ Section of Rheumatology, Department of Pediatrics, College of Medicine and Philippine General Hospital, University of the Philippines Manila \\ ${ }^{3}$ Section of Dermatology, Department of Medicine, College of Medicine and Philippine General Hospital, University of the Philippines Manila
}

\begin{abstract}
Bullous eruptions are rare cutaneous manifestations of systemic lupus erythematosus. We report a case of an 8-yearold Filipino girl with vesiculobullous systemic lupus erythematosus (SLE) and membranous lupus nephritis on kidney biopsy who presented with clinical nephrotic features of generalized edema, proteinuria, hypoalbuminemia and hyperlipidemia. The 2012 Systemic Lupus International Collaborating Clinics (SLICC) classification criteria for SLE were met. Immunohistopathologic examination of the skin lesion revealed a sub-epidermal split with neutrophilic infiltrates along the dermo-epidermal junction, moderate perivascular, periadnexal and interstitial infiltrates composed of predominantly neutrophils with neutrophilic dusts, lymphocytes, plasma cells, rare eosinophils and increased dermal mucin. Direct immunofluorescence showed strong continuous linear IgG deposits along the basement membrane and weak linear IgM and IgA deposition along the basement membrane zone (BMZ). To our knowledge, this is the first report of vesiculobullous SLE in a Filipino child. This case is a rare form of cutaneous lupus in children. Bullous SLE (BSLE) should be considered in the differential diagnosis of children presenting with generalized bullous eruptions.
\end{abstract}

Key Words: Systemic lupus erythematosus, child, membranous lupus nephritis, vesiculobullous, bullous lupus

\section{BACKGROUND}

Cutaneous involvement is seen in up to $85 \%$ of Systemic Lupus Erythematosus (SLE) patients during the course of the disease. ${ }^{1}$ Of the skin manifestations of SLE, vesiculobullous eruption or simply known as bullous SLE (BSLE) is seen in less than $1 \%$ of SLE cases. To date, there are 21 reported cases in the literature from 1979..$^{2-19}$

Of the 357 cases of juvenile SLE and 169 cases of childhood lupus nephritis seen by the section of Pediatric Rheumatology at the Philippine General Hospital (PGH) since 2005, this is the first documented case that presented with generalized vesiculobullous lesions. ${ }^{20}$

\section{CASE PROFILE}

An 8-year-old Filipino girl sought consult at PGH

Corresponding author: Lourdes Paula R. Resontoc, MD Section of Nephrology

Department of Pediatrics

College of Medicine

Philippine General Hospital

University of the Philippines Manila

Taft Avenue, Manila 1000, Philippines

Telephone: +639178309796

Email: Irresontoc@up.edu.ph with a one-month history of generalized edema. She was initially diagnosed with idiopathic nephrotic syndrome and was treated with oral steroids. Two weeks after initial management, the patient reported hair fall, the appearance of rashes on her palms and perioral sores. On follow-up with a private pediatrician, consideration of SLE was entertained. Diagnostics were requested with a significant antinuclear antibody (ANA) value of 28.4 (positive: > 1.0). Oral steroid 
was continued but she was subsequently admitted to PGH due to new reported symptoms of hair fall and rashes. Two days after admission, pruritic vesicular skin eruptions with blister formation were noted which were initially seen on her eyelids, vermillion borders of lips, both ear lobes and later involved the neck, shoulders, trunk and to a lesser extent, the dorsal aspect of the hands and palms, axillae, perineal area and the lower extremities. The review of systems and past medical history were unremarkable. Her family history revealed an older sibling and a maternal aunt with SLE.

On examination, the child was seen with generalized edema and prominent vesicles and tense bullae which were evident at the perioral area extending on the hairline, neck, shoulders and trunk. There were few blisters on the palms and soles as well. Ulcers on the buccal mucosa, inner and outer lips were also noted. Skin fragility and obvious scarring were not noted (Figure 1).

Laboratory tests on admission showed the following results: hemoglobin $89 \mathrm{~g} / \mathrm{L}$, hematocrit 0.27 , total leucocyte count $17.33 \times 10^{9} / \mathrm{L}$ with neutrophil predominance, red blood cell count of $3.26 \times 10^{12} / \mathrm{L}$ and adequate platelet count, erythrocyte sedimentation rate $120 \mathrm{~mm} / \mathrm{hr}$, C3 was $0.8 \mathrm{~g} / \mathrm{L}$ (normal value $0.89-1.87 \mathrm{~g} / \mathrm{L}$ ). There was no antidouble stranded deoxyribonucleic acid (anti-dsDNA) determination available. Serum creatinine was $50 \mathrm{umol} / \mathrm{L}$ with serum albumin of $22 \mathrm{~g} / \mathrm{L}$. Lipid profile showed elevated cholesterol and triglycerides levels at $12.13 \mathrm{mmol} / \mathrm{L}$ and $2.03 \mathrm{mmol} / \mathrm{L}$ respectively. Urinalysis showed specific gravity of 1.023 , albumin $+3, \mathrm{RBC} 38$ per high power field (hpf), WBC 6 per hpf and presence of hyaline cast.
The 24-hour urine protein was $1560 \mathrm{mg} / \mathrm{day} / 1.73 \mathrm{~m}^{2}$. Blood and urine cultures were negative for bacteria. Multinucleated cells were not demonstrated on Tzanck smear. Titers for herpes simplex virus (HSV) 1 and 2 viruses were negative. Varicella $\mathrm{IgG}>150 \mathrm{u} / \mathrm{mL}$ (positive titer: > $12 \mathrm{u} / \mathrm{ml}$ ) but negative for IgM. Echocardiography done showed left ventricular enlargement.Kidney biopsy which was done after $2^{\text {nd }}$ cycle of methylprednisolone pulse therapy and 5 months of mycophenolate mofetil intake showed class $\mathrm{V}$ (membranous) lupus nephritis with mild interstitial fibrosis and tubular atrophy.

Biopsy of a blister showed a sub-epidermal split with neutrophilic infiltrates along the dermo-epidermal junction, moderate perivascular, periadnexal and interstitial infiltrates composed of predominantly neutrophils with neutrophilic dusts, lymphocytes, plasma cells, rare eosinophils and increased dermal mucin. Direct immunofluorescence showed strong continuous linear IgG deposits along the basement membrane and weak linear $\operatorname{IgM}$ and $\operatorname{IgA}$ deposition along the basement membrane zone (BMZ) ( Figure 2). A diagnosis of vesiculobullous SLE was made. Though the initial plan was to start dapsone, the presence of low glucose-6-phosphae dehydrogenase (G6PD) level prompted the use of pulse methylprednisolone therapy (MPPT) and mycophenolate mofetil (MMF) instead with noted signs of improvement (e.g. clearing of vesicles and bullae) which were seen as early as the $1^{\text {st }}$ week from treatment initiation. The blisters and erosions healed without scarring. The SLE nephritis was kept in control with oral prednisone, MMF and hydroxychloroquine.
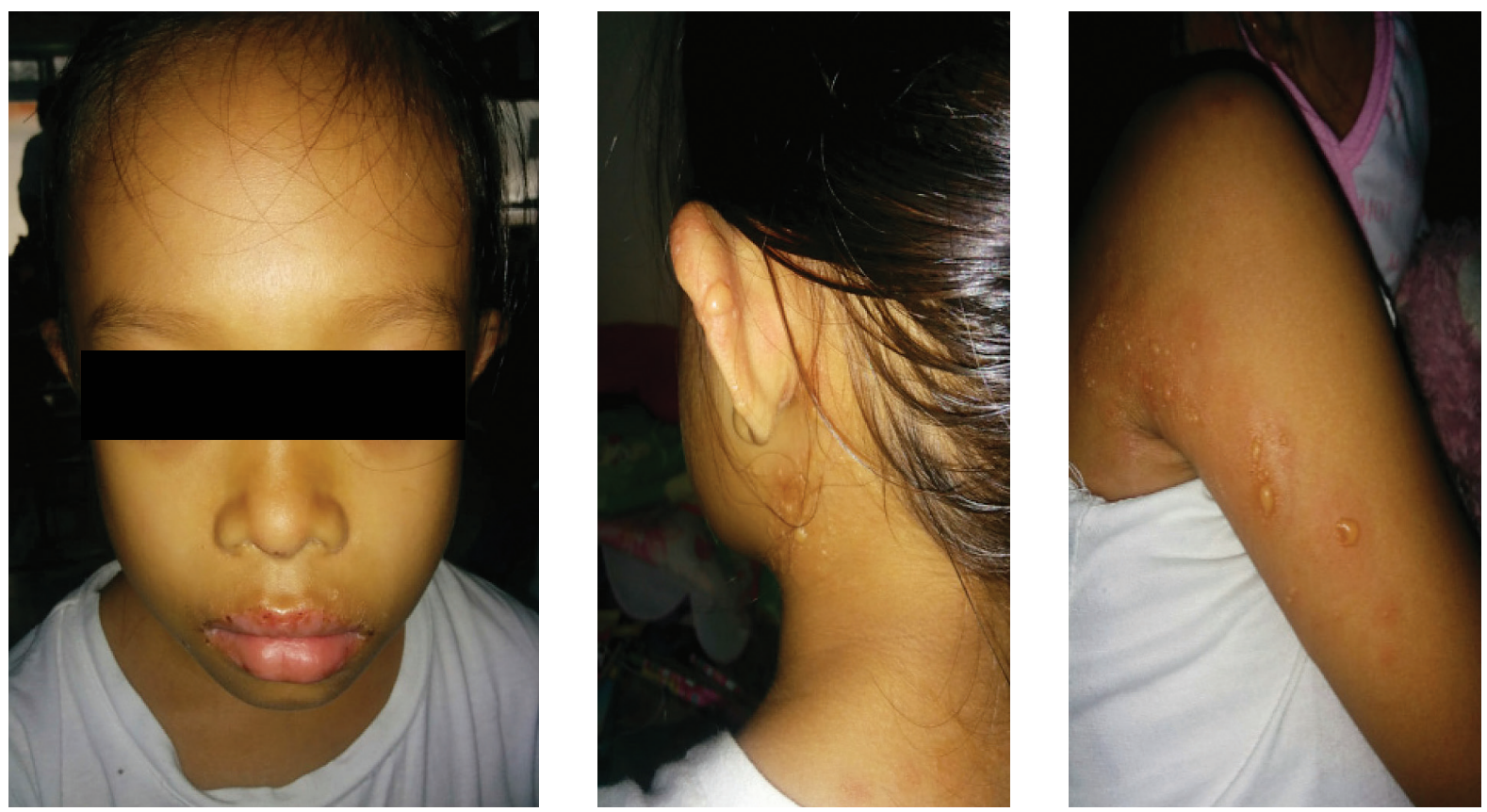

Figure 1. Multiple tense vesicles and bullae filled with clear serous fluid seen on the vermillion border of the lips, pinna of both ears, neck and arms. Nikolsky's sign is negative for all lesions. (Verbal and written consents obtained from parents in the publication of above images). 

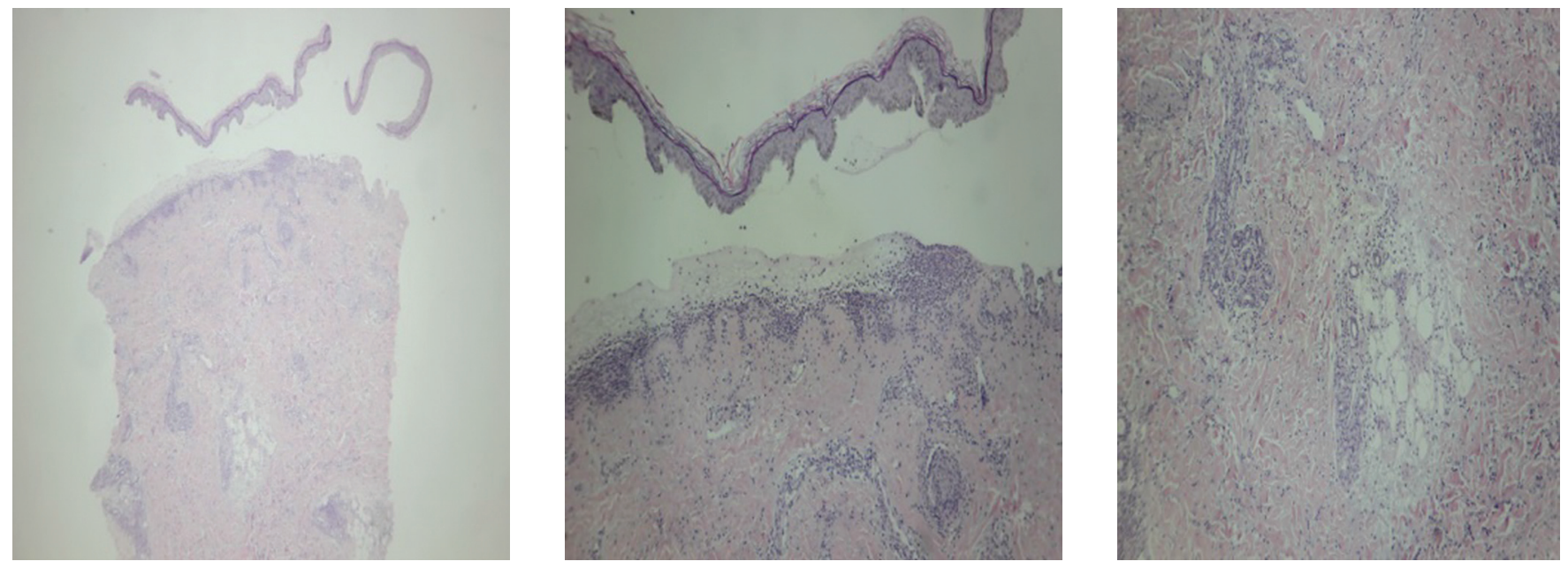

Figure 2. Histopathologic examination revealed a sub-epidermal split with neutrophilic infiltrates along the dermo-epidermal junction, moderate perivascular, periadnexal and interstitial infiltrates composed of predominantly neutrophils with neutrophilic dusts, lymphocytes, plasma cells, rare eosinophils and increased dermal mucin.

\section{DISCUSSION}

The patient in this report was initially treated as a case of idiopathic nephrotic syndrome due to absence of other systemic manifestations. The development of cutaneous manifestations such as rash, oral sores and hair problems with concomitant proteinuria, anemia, an elevated ANA titer and low $\mathrm{C} 3$ value clinched the diagnosis of SLE based on the 2012 SLICC criteria. To add, the kidney biopsy finding of class $\mathrm{V}$ lupus nephritis later confirmed the renal involvement of the said diagnosis.

The appearance of vesiculobullous lesions during the course of patient's illness suggested the possibility of viral exanthem in an immunocompromised individual hence initially prompting work-up for an infectious process. However, results of available viral panels showed negative values making an infectious cause of the lesion unlikely. Bullous dermatoses were then entertained.

The skin biopsy finding of a sub-epidermal split with predominantly neutrophilic infiltrates coupled by the linear $\operatorname{IgG}, \operatorname{IgM}$ and $\operatorname{IgA}$ deposits along the basement membrane on direct immunofluorescence highly suggested the diagnosis of BSLE. Among the differential diagnoses of BSLE include blistering diseases such as bullous pemphigoid, epidermolysis bullosa acquisita, linear IgA dermatosis and dermatitis herpetiformis. ${ }^{16-19,21-22}$

BSLE is a rare form of SLE and the absence of pathognomonic clinical features renders the difficulty in clinching the diagnosis. ${ }^{16-19,21}$ The first diagnostic criteria for BSLE was proposed in 1983 by Camisa and Sharma which include the following: (1) a diagnosis of SLE based upon American Rheumatism Association criteria, (2) vesicles and bullae arising upon but not limited to sun-exposed skin, (3) histopathology compatible with dermatitis herpetiformes, (4) Negative indirect immunofluorescence for circulating BMZ antibodies and (5) direct immunofluorescence of lesional and nonlesional skin revealing linear or granular IgG and/or IgM and often IgA at the BMZ.22-23 The association of BSLE with circulating antibodies directed against type VII collagen led to a later revision of the diagnostic criteria by Gammon et al. ${ }^{24}$ wherein patients with the said antibodies were designated as having Type 1 BSLE while those who did not demonstrate them were labeled with Type 2 BSLE. Clinically, the distinction between the two types is not possible prompting the need for subtyping through immunohistochemistry. 25 Despite progress in the characterization of BSLE as a disease associated with autoimmunity directed towards type VII collagen, evidences remain scarce in establishing whether the collagen subtype is the sole target antigen in the disease. ${ }^{21}$

Affected individuals with BSLE are usually young females with the typical presentation starting in the second decade of life. ${ }^{8,22}$ Of note, only several cases have been documented in the pediatric population with a 5-year-old patient as the youngest patient reported in $2014 .{ }^{16}$

The classic description of tense vesicles and larger bullae may mimic the blistering manifestations of other bullous dermatoses. However, the absence of scarring and mechanical fragility in BSLE sets it apart from other differential diagnoses although pigmentary changes occur in those with darker skin types. ${ }^{25}$

The separation of the epidermis from the dermis at the $\mathrm{BMZ}$ and the predominance of fibrin and neutrophils in the blister cavity are classic histopathologic features of BSLE that are indistinguishable from those of dermatitis herpetiformis. However, the presence of mucin in the reticular dermis as seen in the patient's histopathology provides a distinctive feature of BSLE from other blistering diseases. ${ }^{22}$ Studies have shown that granular pattern of immunoglobulin deposition is seen in $60 \%$ of cases while $40 \%$ is that of a linear pattern. In the patient's direct immunofluorescence result, all 
major immunoglobulins ( $\operatorname{Ig} G, \operatorname{Ig} A, \operatorname{Ig} M)$ are involved in the deposition along the dermo-epidermal junction which is consistent with the available literature wherein $\mathrm{IgG}$ being almost invariably present.

Correlating with the patient's course, the appearance of vesiculobullous lesion occurred concurrently with the SLE nephritis activity as indicated by nephrotic-range proteinuria, generalized edema and low C3 level. Several studies have already described the close temporal relationship of BSLE with an SLE activity hence suggesting a possible association between the two. ${ }^{14,21}$ In a study done in 2001 by Onetti Muda et al., the findings of synthesis and incorporation of type VII collagen, which is not normally present in glomeruli, in areas of glomerular and/or tubular scarring irrespective of the underlying disease confirmed the de novo expression of fibrillary collagens in the diseased renal extracellular matrix. ${ }^{14}$ The simultaneous occurrence of BSLE in a patient with lupus nephritis can then be hypothesized to be secondary to the initiation of antibody production and reaction to the ectopic production and/or deposition of type VII collagen in the kidney during the activity of SLE.

The dramatic therapeutic response of BLSE to dapsone is a prominent distinguishing feature which separates it from other diagnoses, ${ }^{4,14,21}$ The effect of dapsone lies in the inhibition of the functions of neutrophils and complement activation via alternative pathway. ${ }^{26}$ However, pulse methylprednisolone therapy and mycophenolate mofetil were administered due to a documented low G6PD level which provided control of both bullous manifestation and nephritis. The use of high-dose corticosteroids in conjunction with another immunosuppressant has been suggested as an alternative modality whenever flare of SLE coincides with the bullous lesion and whenever dapsone is unavailable or contraindicated. ${ }^{21}$

In summary, we presented an 8-year-old female who developed vesiculobullous skin lesions during active nephritis with clinicoserologic values consistent with BSLE who responded with alternative modality which included MPPT and MMF. BSLE should always be considered in the differential diagnosis in pediatric patients presenting with blistering eruptions.

\section{Statement of Authorship}

All authors have approved the final version submitted.

\section{Author Disclosure}

All authors declared no conflict of interest.

\section{Funding Source}

No funding.

\section{REFERENCES}

1. Rothfield N, Sontheimer R, Bernstein M. Lupus erythematosus: systemic and cutaneous manifestations. Clin Dermatol. 2006; 24(5):348-62 .
2. Jacoby RA, Abraham AA. Bullous dermatosis and systemic lupus erythematosus in a 15-year-old boy. Arch Dermatol. 1979; 115(9): 1094-97.

3. Hall RP, Lawley TJ, Smith HR et al. Bullous eruption of systemic lupus erythematosus: dramatic response to dapsone therapy. Ann Intern Med.1982; 197:165-70.

4. Kettler AH, Bean SF, Duffy JO, et al. Systemic lupus erythematosus presenting as a bullous eruption in a child. Arch Dermatol. 1988; 124(7):1083-87.

5. Roholt NS, Lapiere JC, Wang JI, et al. Localized linear bullous eruption of systemic lupus erythematosus in a child. Pediatr Dermatol. $1995 ; 12: 138-44$.

6. Shirhama S, Furukawa F, Yagi H, et al. Bullous systemic lupus erythematosus: detection of antibodies against noncollagenous domain of type VII collagen. J Am Acad Dermatol. 1998; 38:844-48.

7. Chan LS, Lapier JC, Chen M, et al. Bullous systemic lupus erythematosus with autoantibodies recognizing multiple skin basement membrane components, bullous pemphigoid antigen 1, laminin-5, laminin-6, and type VII collagen. Arch Dermatol. 1999; 135:569-73.

8. Nitta Y, Kawamwa C, Hashimoto T. Vesicullobullous systemic lupus erythematosus: a case with circulating $\operatorname{IgG}$ and $\operatorname{IgA}$ autoantibodies to type VII collagen. J Am Acad Dermatol. 2002; 47(5):283-86.

9. Harris-Stith R, Ericsson QL, Elston DM, et al. Bullous eruption: a manifestation of lupus erythematosus. Cutis. 2003; 72:31-37.

10. Sirka CS, Padhi T, Mohanty P, et al. Bullous systemic lupus erythematosus: response to dapsone in two patients. Indian J Dermatol Venereol Leprol. 2005; 71:54-6.

11. Vijayalakshmi AM, Jayavardhana A. Bullous systemic lupus erythematosus and lupus nephritis in a 10 year old boy. Indian Pediatr. 2007; 44(11): 861-3.

12. Kumar S, Agarwal I. Unusual presentation of childhood systemic lupus erythematosus. Pediatr Rheumatol Online J. 2007; 31(5):20.

13. Tincopa M, Puttgen KB, Sule S, et al. Bullous lupus: An unusual initial presentation of systemic lupus erythematosus in an adolescent girl. Pediatr Dermatol. 2010; 27(4): 373-6.

14. Sáez-de-Ocariz M, Espinosa-Rosales F, López-Corella E, et al. Bullous lesions as manifestation of systemic lupus erythematosus in two Mexican teenagers. Pediatr Rheumatol Online J. 2010; 8:19. doi: 10.1186/1546-0096-8-19.

15. Poojary S, Rais S. Bullous systemic lupus erythematosus with lupus nephritis. A rare case of a subepidermal bullous disorder in a child. Cutis. 2012; 89(1): 17-21.

16. Lourenço DM, Gomes RC, Aikawa NE, et al. Childhood-onset bullous systemic lupus erythematosus. Lupus. 2014; 23(13): 1422-5.

17. Christodoulou G, Powell M, Nguyen VH, et al. An atypical case of bullous systemic lupus erythematosus in a 16-year-old boy. Pediatr Dermatol. 2014; 31(6): 64-6.

18. Kong Y, Lim YL, Chandran NS. Retrospective study on autoimmune blistering disease in paediatric patients. Pediatr Dermatol. 2015; 32(6): 845-52.

19. Maggio MC, Corsello G, Prinzi E, et al. Systemic Lupus Erythematosus and Bullous Pemphigoid with Dramatic Response. Am J Case Rep. 2017; 18: 317-319.

20. UP PGH Section of Pediatric Rheumatology Annual census 20052016. (Unpublished)

21. Fujimoto W1, Hamada T, Yamada J, Matsuura H, Iwatsuki K. Bullous systemic lupus erythematosus as an initial manifestation of SLE. J Dermatol. 2005; 32(12):1021-7

22. Vassileva S. Bullous systemic erythematosus. Clin Dermatol. 2004; 22(2):129-38.

23. Camisa C, Sharma HM. Vesiculobullous systemic lupus erythematosus. J Am Acad Dermatol. 1983 (9):924-33.

24. Gammon WR, Briggaman RA. Bullous SLE: a phenotypically distinctive but immunologically heterogeneous bullous disorder. J Invest Dermatol. 1993; (100):285-345.

25. Sebaratnam D, Muller D. Bullous systemic lupus erythematosus. Dermatol Clin. 2011; (29): 649-53.

26. Duan L, Chen L, Zhong S, et al. Treatment of bullous systemic lupus erythematosus. Journal of Immunology Research. 2014:1-6. 